Australasian Journal of Logic

\title{
A REMARK ON MAKSIMOVA'S VARIABLE SEPARATION PROPERTY IN SUPER-BI-INTUITIONISTIC LOGICS
}

\author{
GUILLERMO BADIA \\ DEPARTMENT OF KNOWLEDGE-BASED MATHEMATICAL SYSTEMS \\ JOHANNES KEPLER UNIVERSITY LINZ \\ GUILLEBADIA89@GMAIL.COM
}

\begin{abstract}
We provide a sufficient frame-theoretic condition for a superbi-intuitionistic logic to have Maksimova's variable separation property. We conclude that bi-intuitionistic logic enjoys the property. Furthermore, we offer an algebraic characterization of the super-bi-intuitionistic logics with Maksimova's property.
\end{abstract}

\section{INTRODUCTION}

Propositional bi-intuitionistic or Heyting-Brouwer logic is a natural expressive extension of propositional intuitionistic logic which has been explored in places like $[9,8]$ and much more recently [5]. It comes from adding a binary connective called subtraction (symbolized as -) to the language of intuitionistic logic while leaving the class of models of intuitionistic logic untouched (hence producing no new validities in the old language of intuitionistic logic). We call a super-bi-intuitionistic logic to any logical calculus which extends axiomatically bi-intuitionistic logic (in the sense that it adds a - possibly emptyset of new validities to the theorems of bi-intuitionistic logic). As a referee points out, it is worth mentioning that our super-bi-intuitionistic logics have received other names in the literature, for instance, "super-HB logics" ([11], p. 356).

In [6], the following two open problems were posed:

(i) Does bi-intuitionistic logic have Maksimova's variable separation property?

(ii) Provide an algebraic characterization of the super-bi-intuitionistic logics with the property.

Maksimova's property is a generalization of Halldén completeness (i.e., the property that if two formulas with no propositional variables in common have countermodels then we must be able to find a countermodel to their disjunction) which was originally introduced and studied (for relevant logics) in [7] (see $\S 3$ ). Borrowing from the simple model-theoretic techniques of [7], we give a positive answer to (i) in $\S 4$. In passing, we establish an analogue of the Australasian Journal of Logic (14:1) 2017, Article no. 3 
main result from [1] on Halldén completeness but for Maksimova's property. Finally, in $\S 5$ we also give an answer to (ii).

\section{Bi-InTUitionistiC LOGIC}

A propositional bi-intuitionistic or Heyting-Brouwer language $L$ contains a list PROP of propositional variables $p, q, r \ldots$, as well as the logical symbols $\perp$, $\vee, \wedge, \Rightarrow$ and new binary connective - dual to $\Rightarrow$.

An algebraic semantics for $L$ is provided by Heyting-Brouwer algebras (also called double Heyting algebras), that is, structures of the form $\langle A, \vee, \wedge, \Rightarrow$ $,-, 0,1\rangle$ where both $\langle A, \vee, \wedge, \Rightarrow, 0,1\rangle$ and $\left\langle A, \vee^{\delta}, \wedge^{\delta},-, 0^{\delta}, 1^{\delta}\right\rangle$ are Heyting algebras, where the dual operation $\delta$ is as usual: $\vee^{\delta}=\wedge, \wedge^{\delta}=\vee, 0^{\delta}=$ $1,1^{\delta}=0 . \operatorname{In}\left\langle A, \vee^{\delta}, \wedge^{\delta}, 0^{\delta}, 1^{\delta}\right\rangle$ the partial order $\leqslant$ (which is defined as always in terms of $\vee$ or $\wedge$ ) is the converse ordering of $\langle A, \vee, \wedge, 0,1\rangle$. The following will hold (for the partial ordering of $\langle A, \vee, \wedge, 0,1\rangle$ ):

$$
\begin{aligned}
& x \wedge y \leqslant z \text { iff } y \leqslant x \Rightarrow z, \\
& x \vee y \geqslant z \text { iff } y \geqslant z-x .
\end{aligned}
$$

The paper [10] is a place where these algebras have been studied. An equational basis for this class of algebras is given by the equational basis for Heyting algebras plus their dual equations for the dual algebra.

A Kripke frame for $L$ is a structure $\mathscr{F}=\langle W, R\rangle$, where $W$ is a non-empty set and $R \subseteq W \times W$ is a partial order. A model $M=\langle\mathscr{F}, V\rangle$, is a frame together with a valuation function $V:$ PROP $\longrightarrow \wp(W)$ satisfying the following closure property:

$$
\text { if } w \in V(p) \text { and } R w u \text { then } u \in V(p) .
$$

We define satisfaction at $w$ in $M$ recursively as follows:

$$
\begin{array}{lll}
M, w \Vdash \perp & \text { never, } & \\
M, w \Vdash p & \text { iff } & w \in V(p), \\
M, w \Vdash(\phi \wedge \psi) & \text { iff } & M, w \Vdash \phi \text { and } M, w \Vdash \psi, \\
M, w \Vdash(\phi \vee \psi) & \text { iff } & M, w \Vdash \phi \text { or } M, w \Vdash \psi, \\
M, w \Vdash \phi \Rightarrow \psi & \text { iff } & \text { for every } a \text { such that } R w a \text {, if } M, a \Vdash \phi \text { then } M, a \Vdash \psi, \\
M, w \Vdash \phi-\psi & \text { iff } & \text { there is } a \text { such that } R a w \text { and } M, a \Vdash \phi \text { while } M, a \Vdash \psi
\end{array}
$$

By bi-intuitionistic logic (which we will abbreviate as BiInt) we will be mean the set of formulas true at every world in all the above defined Kripke models, i.e., the formulas valid in all the above defined frames.

\section{MAKSIMOVA'S PROPERTY}

If $\phi_{1}, \ldots, \phi_{n}$ is some list of formulas, by $\operatorname{Var}\left(\phi_{1}, \ldots, \phi_{n}\right)$ we will mean the collection of propositional variables appearing in any of the $\phi_{i}(1 \leqslant i \leqslant n)$. Australasian Journal of Logic (14:1) 2017, Article no. 3 
Definition 1. A super-bi-intuitionistic logic $\mathbf{L}$ is said to have Maksimova's variable separation property if whenever $\operatorname{Var}\left(\phi_{1}, \phi_{2}\right) \cap \operatorname{Var}\left(\psi_{1}, \psi_{2}\right)=\varnothing$, then

$(*) \phi_{1} \wedge \psi_{1} \Rightarrow \phi_{2} \vee \psi_{2} \in \mathbf{L}$ only if either $\phi_{1} \Rightarrow \phi_{2} \in \mathbf{L}$ or $\psi_{1} \Rightarrow \psi_{2} \in \mathbf{L}$.

Maksimova's property is closely related to the Halldén completeness of a logic. Recall that a logic is said to be Halldén complete if whenever $\operatorname{Var}(\phi) \cap$ $\operatorname{Var}(\psi)=\varnothing$ and $\phi \vee \psi$ is a theorem, then either $\phi$ is a theorem or $\psi$ is a theorem.

For any super-bi-intuitionistic logic L, Maksimova's property implies Halldén completeness. To see this, define $T={ }_{d f} \perp \Rightarrow \perp$, noting that $T \in \mathbf{L}$. Suppose $\phi \vee \psi \in \mathbf{L}$, but then $\top \wedge \top \Rightarrow \phi \vee \psi \in \mathbf{L}$. By Maksimova's property for $\mathbf{L}$, either $\top \Rightarrow \phi \in \mathbf{L}$ or $\top \Rightarrow \psi \in \mathbf{L}$. However, $T \in \mathbf{L}$, so either $\phi \in \mathbf{L}$ or $\psi \in \mathbf{L}{ }^{1}$

\section{Bi-INTUitionistic LOGIC has MAKSIMOVA'S PROPERTY}

We start this section by introducing a notion of $p$-morphism for biintuitionistic frames. This idea is well-known from intuitionistic (and modal) logic (cf. [2], p. 30).

Definition 2. Let $\mathscr{F}_{1}=\left\langle W_{1}, R_{1}\right\rangle, \mathscr{F}_{2}=\left\langle W_{2}, R_{2}\right\rangle$ be a pair of frames. A p-morphism from $\mathscr{F}_{1}$ onto $\mathscr{F}_{2}$ is a surjective map $f: W_{1} \longrightarrow W_{2}$ such that

(i) $R_{1} w v$ only if $R_{2} f(w) f(v)$

(ii) $R_{2} f(w) f(v)$ only if there is $u \in W_{1}$ such that $R_{1} w u$ and $f(u)=f(v)$

(iii) $R_{2} f(v) f(w)$ only if there is $u \in W_{1}$ such that $R_{1} u w$ and $f(u)=f(v)$

THEOREM 1. (p-morphism theorem) Let $f$ be a p-morphism from a frame $\mathscr{F}_{1}$ to a frame $\mathscr{F}_{2}$. Then for any formula $\phi$ of a language $L,\left\langle\mathscr{F}_{1}, V^{\prime}\right\rangle, w \Vdash \phi$ iff $\left\langle\mathscr{F}_{2}, V\right\rangle, f(w) \Vdash \phi$, where for each propositional variable $p$ of $L, f^{-1}(V(p))=$ $V^{\prime}(p)$.

Proof. This is as for Theorem 2.15 from [2]. It proceeds by an easy induction on formula complexity and we leave it to the reader. For the case $\phi \rightarrow \psi$ we appeal to the property (ii) in Definition 2 whereas for $\phi-\psi$ we use the new condition (iii).

Definition 3. A class $K$ of frames is said to be closed under $p$-morphic super-fusion if for any $\mathscr{F}_{1}, \mathscr{F}_{2} \in K$, worlds $w_{1}, w_{1}^{\prime} \in W_{1}, w_{2}, w_{2}^{\prime} \in W_{2}$ such that $R_{1} w_{1} w_{1}^{\prime}$ and $R_{2} w_{2} w_{2}^{\prime}$, there is $\mathscr{F}_{3} \in K$, worlds $w_{3}, w_{3}^{\prime} \in W_{3}$ and $p$ morphisms $f_{1}: W_{3} \longrightarrow W_{1}, f_{2}: W_{3} \longrightarrow W_{2}$ such that $R_{3} w_{3}, w_{3}^{\prime}$ while $f_{1}\left(w_{3}\right)=w_{1}, f_{1}\left(w_{3}^{\prime}\right)=w_{1}^{\prime}, f_{2}\left(w_{3}\right)=w_{2}$ and $f_{2}\left(w_{3}^{\prime}\right)=w_{2}^{\prime}$. In a picture,

\footnotetext{
${ }^{1}$ The Halldén completeness of BiInt is proven via a clever syntactic argument in [6].
} Australasian Journal of Logic (14:1) 2017, Article no. 3 


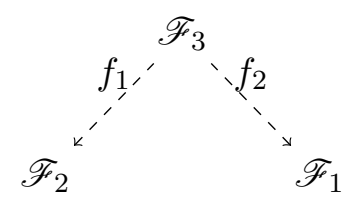

The following result is the analogue of Theorem 1 from [1].

THEOREM 2. Any super-bi-intuitionistic logic $\mathbf{L}$ determined by a class of frames closed under p-morphic super-fusion enjoys Maksimova's variable separation property.

Proof. Let $\operatorname{Var}\left(\phi_{1}, \phi_{2}\right) \cap \operatorname{Var}\left(\psi_{1}, \psi_{2}\right)=\varnothing$. Let $L_{\phi_{1}, \phi_{2}}$ and $L_{\psi_{1}, \psi_{2}}$ be the languages with only the propositional variables in $\operatorname{Var}\left(\phi_{1}, \phi_{2}\right)$ and $\operatorname{Var}\left(\psi_{1}, \psi_{2}\right)$ respectively. We argue for the contrapositive. Thus suppose $\phi_{1} \Rightarrow \phi_{2} \notin \mathbf{L}$ and $\psi_{1} \Rightarrow \psi_{2} \notin \mathbf{L}$. Hence, there are models $\left\langle\mathscr{F}_{1}, V_{1}\right\rangle,\left\langle\mathscr{F}_{2}, V_{2}\right\rangle$ (for $L_{\phi_{1}, \phi_{2}}$ and $L_{\psi_{1}, \psi_{2}}$ respectively) and worlds $w_{1} \in W_{1}, w_{2} \in W_{2}$ such that such that $\left\langle\mathscr{F}_{1}, V_{1}\right\rangle, w_{1} \Downarrow \phi_{1} \Rightarrow \phi_{2}$ and $\left\langle\mathscr{F}_{2}, V_{2}\right\rangle, w_{2} \Vdash \psi_{1} \Rightarrow \psi_{2}$. Consequently, there is $w_{1}^{\prime}$ such that $R_{1} w_{1} w_{1}^{\prime},\left\langle\mathscr{F}_{1}, V_{1}\right\rangle, w_{1}^{\prime} \Vdash \phi_{1}$ while $\left\langle\mathscr{F}_{1}, V_{1}\right\rangle, w_{1}^{\prime} \Vdash \phi_{2}$, and $w_{2}^{\prime}$ such that $R_{2} w_{2} w_{2}^{\prime},\left\langle\mathscr{F}_{2}, V_{2}\right\rangle, w_{2}^{\prime} \Vdash \psi_{1}$ while $\left\langle\mathscr{F}_{2}, V_{2}\right\rangle, w_{2}^{\prime} \Vdash \psi_{2}$.

By the closure under $p$-morphic super-fusion assumption, we see that there must be a frame $\mathscr{F}_{3}$ for $\mathbf{L}$, worlds $w_{3}, w_{3}^{\prime} \in W_{3}$ and $p$-morphisms $f_{1}: W_{3} \longrightarrow W_{1}, f_{2}: W_{3} \longrightarrow W_{2}$ such that $R_{3} w_{3}, w_{3}^{\prime}$ while $f_{1}\left(w_{3}\right)=$ $w_{1}, f_{1}\left(w_{3}^{\prime}\right)=w_{1}^{\prime}, f_{2}\left(w_{3}\right)=w_{2}$ and $f_{2}\left(w_{3}^{\prime}\right)=w_{2}^{\prime}$. Recalling our assumption that $\operatorname{Var}\left(\phi_{1}, \phi_{2}\right) \cap \operatorname{Var}\left(\psi_{1}, \psi_{2}\right)=\varnothing$, we might consider a valuation $V_{3}$ on $\mathscr{F}_{3}$ such that $V_{3}(p)=f_{1}^{-1}\left(V_{1}(p)\right)$ for $p \in L_{\phi_{1}, \phi_{2}}$ and $V_{3}(p)=f_{2}^{-1}\left(V_{2}(p)\right)$ for $p \in L_{\psi_{1}, \psi_{2}}$. By properties of $p$-morphisms and the closure properties of $V_{1}, V_{2}$, we have that $V_{3}$ must have the required closure property to qualify as a valuation.

According to the $p$-morphism theorem we have that for any formulas $\theta, \sigma$ of $L_{\phi_{1}, \phi_{2}}$ and $L_{\psi_{1}, \psi_{2}}$ respectively,

$$
\begin{aligned}
& \left\langle\mathscr{F}_{3}, V_{3}\right\rangle, w \Vdash \theta \text { iff }\left\langle\mathscr{F}_{1}, V_{1}\right\rangle, f_{1}(w) \Vdash \theta, \\
& \left\langle\mathscr{F}_{3}, V_{3}\right\rangle, w \Vdash \sigma \text { iff }\left\langle\mathscr{F}_{2}, V_{2}\right\rangle, f_{2}(w) \Vdash \sigma .
\end{aligned}
$$

Hence, $\left\langle\mathscr{F}_{3}, V_{3}\right\rangle, w_{3}^{\prime} \Vdash \phi_{1}$ and $\left\langle\mathscr{F}_{3}, V_{3}\right\rangle, w_{3}^{\prime} \Vdash \psi_{1}$, so $\left\langle\mathscr{F}_{3}, V_{3}\right\rangle, w_{3}^{\prime} \Vdash \phi_{1} \wedge \psi_{1}$. On the other hand, $\left\langle\mathscr{F}_{3}, V_{3}\right\rangle, w_{3}^{\prime} \Downarrow \phi_{2}$ and $\left\langle\mathscr{F}_{3}, V_{3}\right\rangle, w_{3}^{\prime} \Downarrow \psi_{2}$, so $\left\langle\mathscr{F}_{3}, V_{3}\right\rangle, w_{3}^{\prime} \|$ $\phi_{2} \vee \psi_{2}$. But then $\left\langle\mathscr{F}_{3}, V_{3}\right\rangle, w_{3} \Vdash \phi_{1} \wedge \psi_{1} \Rightarrow \phi_{2} \vee \psi_{2}$, so $\phi_{1} \wedge \psi_{1} \Rightarrow \phi_{2} \vee \psi_{2} \notin \mathbf{L}$ as desired.

Next we present a rather standard model-theoretic construction (see, for instance, [3], p. 413) which becomes very useful in arguments where we would like to glue two models together while preserving some desirable features of both.

Australasian Journal of Logic (14:1) 2017, Article no. 3 
Definition 4. Let $\mathscr{F}_{1}=\left\langle W_{1}, R_{1}\right\rangle$ and $\mathscr{F}_{2}=\left\langle W_{2}, R_{2}\right\rangle$ be two frames (which are nothing but structures in the usual sense). We can assume that $W_{1} \cap$ $W_{2}=\varnothing$. The product of $\mathscr{F}_{1}$ and $\mathscr{F}_{2}$ will be the structure $\mathscr{F}_{1} \times \mathscr{F}_{2}=$ $\left\langle W_{1} \times W_{2}, R_{1 \times 2}\right\rangle$ where:

$$
R_{1 \times 2}\langle a, b\rangle\langle c, d\rangle \text { iff } R_{1} a c \text { and } R_{2} b d \text {. }
$$

Note that $R_{1 \times 2}$ is a partial order. This follows since the fact that $R_{1 \times 2}$ is a partial order is expressible by a Horn theory. Recall that a Horn fomula is a first order formula of the form $Q_{0} x_{0}, \ldots, Q_{n} x_{n} \bigwedge_{j<k} \phi_{j}$, where $Q_{i}(i \leqslant n)$ is either $\exists$ or $\forall$ and $\phi_{j}$ is a basic horn formula, that is, a formula of the form $\wedge \Theta \supset \chi$ where $\Theta$ is a finite set of atomic formulas (including the empty set, in which case the formula is just $\chi$ ) and $\chi$ is either atomic or a contradiction. But then the theory with the sentences $\forall x, y, z(R x y \wedge R y z \supset R x z), \forall x(R x x)$ and $\forall x, y(R x y \wedge R y x \supset x=y)$ is Horn. So, by Corollary 9.1.6 from [3], $\mathscr{F}_{1} \times \mathscr{F}_{2}$ must satisfy such theory.

Proposition 3. Any super-bi-intuitionistic logic $\mathbf{L}$ determined by a class of frames axiomatizable by a set of Horn sentences (in the signature containing only a binary predicate $R$ for the accessibility relation of the frames) is closed under p-morphic super-fusion.

Proof. Using Corollary 9.1.6 from [3] again, classes of frames axiomatized by a set of Horn sentences are closed under the formation of products. It suffices to check that the projection map is a $p$-morphism.

We are finally in a position to establish the following result.

COROllary 4. BiInt has Maksimova's variable separation property.

Proof. Immediate from Theorem 2 and Proposition 3 together with the fact that BiInt is determined by a class of frames axiomatizable by a Horn theory.

To end this section, let us look at another example of a super-biintuitionistic logic with Maksimova's property. Consider the axiom $(p \rightarrow \perp) \vee$ $((p \rightarrow \perp) \rightarrow \perp)$, called the weak law of the excluded middle (wem). Proposition 2.37 from [2] establishes that wem corresponds to the frame condition $\forall x, y, z(R x y \wedge R x z \supset \exists u(R y u \wedge R z u))$. Now, by standard first order manipulations, we can push the existential quantifier out in the previous formula to get the equivalent form: $\forall x, y, z \exists u(R x y \wedge R x z \supset R y u \wedge R z u)$. In turn, the latter formula is equivalent to $\forall x, y, z \exists u((R x y \wedge R x z \supset R y u) \wedge(R x y \wedge R x z \supset R z u))$, which is a Horn formula. So, from Theorem 2 and Proposition 3 again, we see that BiInt plus the axiom wem is a super-bi-intuitionistic logic with Maksimova's property. 


\section{Algebraic Characterization}

We characterize the super-bi-intuitionitic logics having Maksimova's property by means of a result entirely analogous to Theorem 5.32 from [4] (indeed the proof is essentially the same but we include it for completeness). Notation is very much as in [4], except that given an algebra $\mathbf{A}$, we use $\operatorname{dom}(\mathbf{A})$ to denote the domain of $\mathbf{A}$. Given a super-bi-intuitionistic logic $\mathbf{L}$, the symbol $\mathrm{V}(\mathbf{L})$ denotes the variety corresponding to $\mathbf{L}$. Given a set of propositional variables $X$, by $\mathbf{F m}(X)$ we denote the set of bi-intuitionistic formulas which can be built from $X$. Given a set of equations $X$, by $\operatorname{Mod}(X)$ we will mean all the structures satisfying $X$. Finally given a collection of formulas $\mathbf{F m}(Y)$ based on a list of propositional variables $Y$ and a logic $\mathbf{L}$, by $\mathbf{F m}(Y) / \equiv_{\mathbf{L}}$ we denote the standard Lindenbaum algebra of $\mathbf{L}$.

Definition 5. Let $\mathbf{C}_{\mathbf{1}}, \mathbf{C}_{\mathbf{2}}, \mathbf{C}$ be algebras such that $\mathbf{C}_{\mathbf{1}}, \mathbf{C}_{\mathbf{2}} \subseteq \mathbf{C}$. We say that $\mathbf{C}_{\mathbf{1}}, \mathbf{C}_{\mathbf{2}}$ form a strongly well-connected pair of $\mathbf{C}$ if for arbitrary $a_{1}, a_{2} \in$ $\operatorname{dom}\left(\mathbf{C}_{\mathbf{1}}\right)$ and $b_{1}, b_{2} \in \operatorname{dom}\left(\mathbf{C}_{\mathbf{2}}\right)$, if $a_{1} \wedge_{\mathbf{C}} b_{1} \leqslant a_{2} \vee_{\mathbf{C}} b_{2}$ in $\mathbf{C}$ then either $a_{1} \leqslant a_{2}$ or $b_{1} \leqslant b_{2}$ in $\mathbf{C}$.

TheOREm 5. For any super-bi-intuitionistic logic $\mathbf{L}$, the following are equivalent:

(i) L has Maksimova's variable separation property.

(ii) For any two non-degenerate $H$ - $B$ algebras $\mathbf{A}, \mathbf{B} \in \mathrm{V}(\mathbf{L})$ there are $H-B$ algebras $\mathbf{C}_{\mathbf{1}}, \mathbf{C}_{\mathbf{2}}, \mathbf{C} \in \mathrm{V}(\mathbf{L}), \mathbf{C}_{\mathbf{1}}, \mathbf{C}_{\mathbf{2}} \subseteq \mathbf{C}$ such that $\mathbf{C}_{\mathbf{1}}$ and $\mathbf{C}_{\mathbf{2}}$ form a strongly well-connected pair of $\mathbf{C}$ and surjective homomorphisms $h$ : $\operatorname{dom}\left(\mathbf{C}_{\mathbf{1}}\right) \longrightarrow \operatorname{dom}(\mathbf{A}), j: \operatorname{dom}\left(\mathbf{C}_{\mathbf{2}}\right) \longrightarrow \operatorname{dom}(\mathbf{B})$. In a picture,

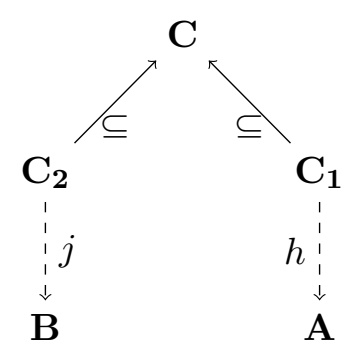

Proof. $(i) \longrightarrow($ ii $)$ : Take any two non-degenerate H-B algebras $\mathbf{A}, \mathbf{B} \in \mathrm{V}(\mathbf{L})$. Consider two disjoint sets of variables $X, Y$ with cardinality greater than or equal to $|\operatorname{dom}(\mathbf{A})|$ and $|\operatorname{dom}(\mathbf{B})|$ respectively. Let $Z=X \cup Y$. For a set of variables $V$, the relation $\equiv_{\mathbf{L}}$ holds between any two $\phi, \psi \in \mathbf{F} \mathbf{m}(V)$ iff $\phi \Rightarrow \psi, \psi \Rightarrow \phi \in \mathbf{L}$. One can see that $\equiv_{\mathbf{L}}$ is a fully invariant congruence relation on $\mathbf{F m}(V)$. Put $\mathbf{C}_{\mathbf{1}}=\mathbf{F} \mathbf{m}(X) / \equiv_{\mathbf{L}}, \mathbf{C}_{\mathbf{2}}=\mathbf{F} \mathbf{m}(Y) / \equiv_{\mathbf{L}}$, and $\mathbf{C}=$ $\mathbf{F m}(Z) / \equiv_{\mathbf{L}}$. Clearly, $\mathbf{C}_{\mathbf{1}}, \mathbf{C}_{\mathbf{2}} \subseteq \mathbf{C}$. $\mathbf{C}_{\mathbf{1}}$ is a free algebra generated by $X$ in the variety $\operatorname{Mod}\left(\left\{\phi=\psi: \phi \equiv_{\mathbf{L}} \psi\right\}\right)$ and similarly with $\mathbf{C}_{\mathbf{2}}, Y, \mathbf{C}$ and $Z$. Now, if $\mathbf{D} \in \mathrm{V}(\mathbf{L})$, and $\phi \equiv_{\mathbf{L}} \psi$, then $\mathbf{D} \vDash(\phi \Rightarrow \psi \wedge \psi \Rightarrow \phi)=1$, Australasian Journal of Logic (14:1) 2017, Article no. 3 
so $\mathbf{D} \vDash \phi=\psi$, i.e., $\mathbf{D} \in \operatorname{Mod}\left(\left\{\phi=\psi: \phi \equiv_{\mathbf{L}} \psi\right\}\right)$. On the other hand if $\mathbf{D} \in \operatorname{Mod}\left(\left\{\phi=\psi: \phi \equiv_{\mathbf{L}} \psi\right\}\right)$, since $\phi \in \mathbf{L}$ iff $\phi \equiv_{\mathbf{L}} 1, \mathbf{D} \in \mathrm{V}(\mathbf{L})$. Hence, $\operatorname{Mod}\left(\left\{\phi=\psi: \phi \equiv_{\mathbf{L}} \psi\right\}\right)=\mathrm{V}(\mathbf{L})$. Since $\mathbf{C}_{\mathbf{1}}, \mathbf{C}_{\mathbf{2}}$ are free, any surjective maps $h_{0}: X \longrightarrow \operatorname{dom}(\mathbf{A}), j_{0}: Y \longrightarrow \operatorname{dom}(\mathbf{B})$ can be extended to surjective homomorphisms $h: \operatorname{dom}\left(\mathbf{C}_{\mathbf{1}}\right) \longrightarrow \operatorname{dom}(\mathbf{A})$ and $j: \operatorname{dom}\left(\mathbf{C}_{\mathbf{2}}\right) \longrightarrow \operatorname{dom}(\mathbf{B})$ respectively.

All that is left is to show that $\mathbf{C}_{\mathbf{1}}$ and $\mathbf{C}_{\mathbf{2}}$ form a strongly well-connected pair. Take arbitrary $\phi_{1} / \equiv_{\mathbf{L}}, \phi_{2} / \equiv_{\mathbf{L}} \in \mathbf{C}_{\mathbf{1}}$ and $\psi_{1} / \equiv_{\mathbf{L}}, \psi_{2} / \equiv_{\mathbf{L}} \in \mathbf{C}_{\mathbf{2}}$. Suppose that $\phi_{1} / \equiv_{\mathbf{L}} \wedge_{\mathbf{C}} \psi_{1} / \equiv_{\mathbf{L}} \leqslant \phi_{1} / \equiv_{\mathbf{L}} \vee_{\mathbf{C}} \psi_{1} / \equiv_{\mathbf{L}}$ in $\mathbf{C}$, but then $\phi_{1} \wedge \psi_{1} \Rightarrow$ $\phi_{2} \vee \psi_{2} \in \mathbf{L}$. Since $\mathbf{L}$ has Maksimova's variable separation property and $\operatorname{Var}\left(\phi_{1}, \phi_{2}\right) \cap \operatorname{Var}\left(\psi_{1}, \psi_{2}\right)=\varnothing$, either $\phi_{1} \Rightarrow \phi_{2} \in \mathbf{L}$ or $\psi_{1} \Rightarrow \psi_{2} \in \mathbf{L}$. This means that either $\phi_{1} / \equiv_{\mathbf{L}} \leqslant \phi_{2} / \equiv_{\mathbf{L}}$ or $\psi_{1} / \equiv_{\mathbf{L}} \leqslant \psi_{2} / \equiv_{\mathbf{L}}$ in $\mathbf{C}$. Thus, $\mathbf{C}_{\mathbf{1}}$ and $\mathbf{C}_{\mathbf{2}}$ form a strongly well-connected pair.

$($ ii $) \longrightarrow(i)$ : Assume that $\operatorname{Var}\left(\phi_{1}, \phi_{2}\right) \cap \operatorname{Var}\left(\psi_{1}, \psi_{2}\right)=\varnothing$ and $\phi_{1} \Rightarrow \phi_{2}, \psi_{1} \Rightarrow$ $\psi_{2} \notin \mathbf{L}$. Hence, there are non-degenerate algebras $\mathbf{A}, \mathbf{B} \in \mathrm{V}(\mathbf{L})$, and valuation maps $f: \mathbf{F m}\left(\operatorname{Var}\left(\phi_{1}, \phi_{2}\right)\right) \longrightarrow \operatorname{dom}(\mathbf{A}), g: \mathbf{F m}\left(\operatorname{Var}\left(\psi_{1}, \psi_{2}\right)\right) \longrightarrow \operatorname{dom}(\mathbf{B})$ such that $f\left(\phi_{1}\right) \$_{\mathbf{A}} f\left(\phi_{2}\right)$ and $g\left(\psi_{1}\right) \$_{\mathbf{A}} g\left(\psi_{2}\right)$. By assumption (ii), there are are H-B algebras $\mathbf{C}_{\mathbf{1}}, \mathbf{C}_{\mathbf{2}}, \mathbf{C} \in V(\mathbf{L}), \mathbf{C}_{\mathbf{1}}, \mathbf{C}_{\mathbf{2}} \subseteq \mathbf{C}$ such that $\mathbf{C}_{\mathbf{1}}$ and $\mathbf{C}_{2}$ form a strongly well-connected pair and surjective homomorphisms $h$ : $\operatorname{dom}\left(\mathbf{C}_{\mathbf{1}}\right) \longrightarrow \operatorname{dom}(\mathbf{A}), j: \operatorname{dom}\left(\mathbf{C}_{\mathbf{2}}\right) \longrightarrow \operatorname{dom}(\mathbf{B})$. We take any valuation $k: \mathbf{F m}\left(\operatorname{Var}\left(\phi_{1}, \phi_{2}\right) \cup \operatorname{Var}\left(\psi_{1}, \psi_{2}\right)\right) \longrightarrow \mathbf{C}$ such that $k(p) \in h^{-1} \circ f(p)$ for $p \in \operatorname{Var}\left(\phi_{1}, \phi_{2}\right)$ and $k(p) \in j^{-1} \circ g(p)$ for $p \in \operatorname{Var}\left(\psi_{1}, \psi_{2}\right)$. Observe that for any formula $\phi$ with variables in $\operatorname{Var}\left(\phi_{1}, \phi_{2}\right), h \circ k(\phi)=f(\phi)$ and for any formula $\psi$ with variables in $\operatorname{Var}\left(\psi_{1}, \psi_{2}\right), j \circ k(\psi)=g(\psi)$. This can be proven by induction on formula complexity, using the facts that $h, j, f, g, k$ are homomorphisms in the inductive hypothesis. Then, $h \circ k\left(\phi_{1}\right) \$_{\mathbf{A}} h \circ k\left(\phi_{2}\right)$ and $j \circ k\left(\psi_{1}\right) \$_{\mathbf{A}}$ $j \circ k\left(\psi_{2}\right)$. Since $h, j$ are surjective homomorphisms and positive first order formulas are preserved under surjective homomorphisms, $k\left(\phi_{1}\right) \$ \mathbf{C}_{\mathbf{1}} k\left(\phi_{2}\right)$ and $k\left(\psi_{1}\right) \hbar_{\mathbf{C}_{\mathbf{2}}} k\left(\psi_{2}\right)$, so since $\mathbf{C}_{\mathbf{1}}, \mathbf{C}_{\mathbf{2}} \subseteq \mathbf{C}, k\left(\phi_{1}\right) \ddagger_{\mathbf{C}} k\left(\phi_{2}\right)$ and $k\left(\psi_{1}\right) \aleph_{\mathbf{C}} k\left(\psi_{2}\right)$. Given that $\mathbf{C}_{\mathbf{1}}, \mathbf{C}_{\mathbf{2}}$ form a well-connected pair, $k\left(\phi_{1}\right), k\left(\phi_{2}\right) \in \operatorname{dom}\left(\mathbf{C}_{\mathbf{1}}\right)$ and $k\left(\psi_{1}\right), k\left(\psi_{2}\right) \in \operatorname{dom}\left(\mathbf{C}_{2}\right)$, we have that $k\left(\phi_{1}\right) \wedge_{\mathbf{C}} k\left(\psi_{1}\right) \aleph_{\mathbf{C}} k\left(\phi_{2}\right) \vee_{\mathbf{C}} k\left(\psi_{2}\right)$. Since $k$ is a homomorphism, $k\left(\phi_{1} \wedge \psi_{1}\right) \$_{\mathbf{C}} k\left(\phi_{2} \vee \psi_{2}\right)$. But this means that $\phi_{1} \wedge \psi_{1} \Rightarrow \phi_{2} \vee \psi_{2} \notin \mathbf{L}$ as desired.

\section{Conclusion}

Any super-bi-intuitionistic logic determined by a class of frames closed under p-morphic super-fusion has Maksimova's property. In particular, biintuitionistic logic satisfies this condition. Moreover, a super-bi-intuitionistic logic has Maksimova's property iff any two non-degenerate algebras in the variety of the logic are homomorphic images of two algebras in that variety forming a strongly well-connected pair. The problem of finding other (more tailored to this context) algebraic characterizations remains open, though. 


\section{ACKNOWLEDGMENTS}

We are quite grateful to an anonymous referee for the AJL who pointed out some important errors in a previous version of this paper. Their comments helped greatly to improve the article. We also wish to thank the organizers of the Frontiers of Non-Classicality conference in January 2016 at Auckland, Zach Weber, Patrick Girard and Maarten McKubre-Jordens for a rather stimulating event.

\section{REFERENCES}

[1] J. van Benthem and I. L. Humberstone. Halldén-completeness by gluing of Kripke frames. Notre Dame Journal of Formal Logic, 24 (4): 426-430 (1983) .

[2] A. Chagrov and M. Zakharyaschev. Modal Logic, Clarendon Press (1997).

[3] W. Hodges. Model Theory. Cambridge (1993).

[4] N. Galatos, P. Jipsen, T. Kowalski and H. Ono. Residuated Lattices: An Algebraic Glimpse at Substructural Logics, Elsevier (2007).

[5] R. Goré and L. Postniece. Combining Derivations and Refutations for Cut-free Completeness in Bi-intuitionistic Logic. Journal of Logic and Computation, 20 (1): 233-260 (2010)

[6] T. Kowalski and H. Ono. Analytic cut and interpolation for bi-intuitionistic logic, manuscript.

[7] L. Maksimova. The principle of separation of variables in propositional logics. Algebra $i$ Logika, 15: 168-184 (1976).

[8] C. Rauszer. A formalization of the propositional calculus in H-B logic. Studia Logica, 33 : 23-34 (1974).

[9] C. Rauszer. Model Theory for an Extension of Intuitionistic Logic. Studia Logica, 36: 73-87 (1977).

[10] H. P. Sankappanavar. Heyting algebras with dual pseudocomplementation. Pacific Journal of Mathematics, 117 (2): 405-415 (1985).

[11] F. Wolter. On logics with coimplication. Journal of Philosophical Logic, 27 (4): 353-387 (1998).

Australasian Journal of Logic (14:1) 2017, Article no. 3 